\title{
Navigation of an Autonomous Underwater Vehicle in a Mobile Network
}

\author{
Nuno Santos, Aníbal Matos and Nuno Cruz \\ Faculdade de Engenharia da Universidade do Porto \\ Instituto de Sistemas e Robótica - Porto \\ Rua Dr ${ }^{\circ}$ Roberto Frias, 4200-465 Porto, Portugal \\ \{nunols,anibal,nacruz\}@fe.up.pt
}

\begin{abstract}
This work addresses the implementation of the navigation, guidance and control of autonomous underwater vehicles on mobile acoustic networks. After a theoretical discussion of the algorithms required for such operation, we present results from simulation and real experiments that validate the proposed solutions.
\end{abstract}

\section{INTRODUCTION}

One of the major limitations of underwater autonomous robotic technologies is the need to moor an acoustic navigation network in the operation area. The use of acoustic mobile networks allows the overcoming of this limitation, leading to a significant reduction in the operational costs associated to the execution of missions.

The quality of the acoustic positioning depends on the network geometry, as discussed in [1]. Positioning errors are minimized by the proper choice of beacon locations, by assuring direct paths between the vehicle and the beacons and avoiding reflections of acoustic signals. Since acoustic signals propagate at a relatively low speed, it is convenient to have small distances between the beacons and the vehicle, to guarantee a high update rate. At the same time, acoustic signals rapidly attenuate with distance. For all these reasons, the operation of underwater vehicles in wide areas traditionally requires the mooring of a large number of acoustic beacons. This increases both the direct operational cost and the mission setup times.

Mobile acoustic networks, composed simply by two moving beacons, are a promising alternative to conventional moored infrastructures, allowing for the reduction of operational costs and making the operation area virtually unbounded. Furthermore, these networks introduce new interesting concepts concerning underwater vehicle missions.

This paper is organized as follows: section II presents the characteristics of the vehicles used to implement the mobile network; then, in section III, the concepts and algorithms required to implement navigation, guidance and control on a moving network are introduced; sections IV and V present, respectively, simulation and experimental results for different scenarios.

\section{EXPERIMENTAL SETUP}

In this work we address a mission on a mobile network involving two autonomous surface crafts (ASC), which carry the acoustic beacons, and an autonomous underwater vehicle (AUV) navigating in that network. Besides, simple acoustic signals used for long baseline (LBL) positioning, we do not rely on any other communication mechanism with the AUV during the execution of a mission. A typical scenario (Fig. 1) where moving beacons greatly decrease operational costs is the bathymetric data collection in rivers with winding margins, covering a few kilometers with no additional resources.

For demonstration purposes we considered a simplified setup with a moored buoy and a moving beacon carried by the Zarco ASC (Fig. 2). This setup also included the MARES AUV (Fig. 3),

Zarco is a small craft developed at Porto University with the capability of transporting payload equipment. It is prepared to perform pre-programmed or real time defined missions [2]. MARES (Modular Autonomous Robot for Environment Sampling) is a small size AUV, configured to carry a wide variety of oceanographic sensors [3]. Its most distinctive feature is the capability of operating at zero forward speed, which is assured by four independent thrusters, two for the horizontal plane motion and the other two for depth and pitch control.

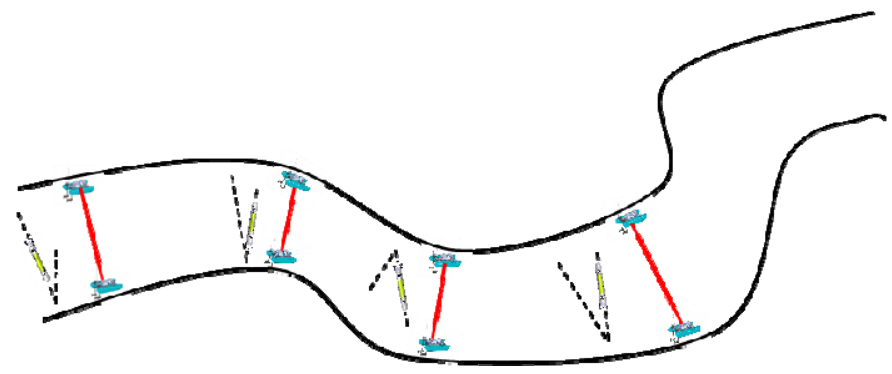

Figure 1. Typical mission with moving acoustic network. 


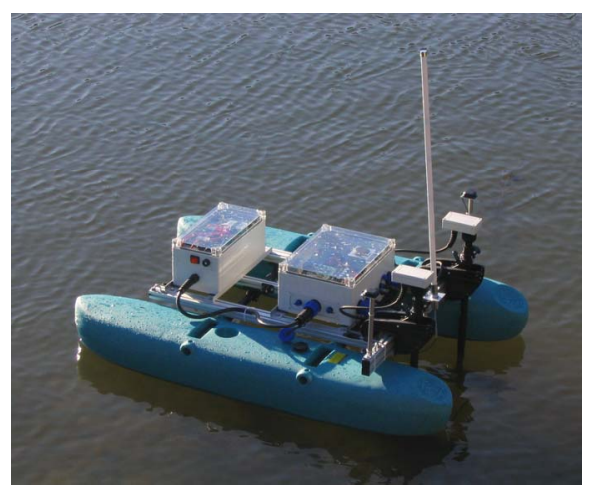

Figure 2. Zarco autonomous surface craft.



Figure 3. MARES autonomous underwater vehicle.

\section{MOBILE NETWORK OPERATION}

The main differences between the operation of an AUV in a fixed and in a mobile acoustic network are the positioning problem, the guidance and control algorithms and the way autonomous missions are programmed.

In a fixed network the vehicle can calculate its global position knowing the global positions of the beacons and its position relative to the beacons. This relative position depends on the distances between the AUV and the beacons (given by travel time measurements of acoustic signals) and also on the distance between the beacons, known as the baseline length, which is an a priori known value.

In a mobile network two issues arise. In the first place, the position of the beacons may change in time and vehicle does not know such variation, which prevents the AUV from determining its global position in real time. Secondly, as the beacons positions vary in time, the baseline length may also change. Therefore, to determine its position relative to the beacons, the AUV must resort to some procedure to estimate the baseline length [4].

The solution proposed here to calculate the baseline length is to use, at least, one additional acoustic signal, as shown in Fig. 4. Besides the two interrogation/reply pairs already used in a two beacon fixed network, one the beacons, number 2 in the example, has to be configured to send a new signal whenever it detects the reply to the vehicle from the other beacon. With respect to this figure, the baseline length can be computed by

$$
b=\frac{t_{4}-t_{0}}{2} \cdot c-d_{B 1}-d_{B 2}
$$

where $c$ is the sound speed in the water, and $d_{B 1}$ and $d_{B 2}$ are distances between the vehicle and the beacons (see [5] for more details).



Figure 4. Transmission / detection communication sequence.

To determine the $x^{\prime}-y^{\prime}$ position relative to the beacons we consider a moving reference frame with the origin on beacon 1 and such that the position of beacon 2 in that frame is given by $(0, b)$, as shown in Fig. 5. It is straightforward to conclude that the relative AUV position can then be computed as

$$
\begin{aligned}
& x^{\prime}=\sqrt{d_{B 1}{ }^{2}-y^{\prime 2}} \\
& y^{\prime}=\frac{d_{B 1}{ }^{2}-d_{B 2}{ }^{2}+b^{2}}{2 b}
\end{aligned}
$$

When operating in a mobile network, the AUV can only determine its position in a mobile reference frame. Therefore, for such operations, the AUV mission has to be programmed in that mobile frame [4]. Only at the end of the mission, and using information about the evolution of the acoustic beacons, can the AUV trajectory be determined in a global Earth fixed frame, according to

$$
\begin{aligned}
& x=x_{B 1}+x^{\prime} \cdot \cos \left(\varphi_{\text {frame }}\right)-y^{\prime} \cdot \sin \left(\varphi_{\text {frame }}\right) \\
& y=y_{B 1}+x^{\prime} \cdot \sin \left(\varphi_{\text {frame }}\right)+y^{\prime} \cdot \cos \left(\varphi_{\text {frame }}\right)
\end{aligned}
$$

where $\varphi_{\text {frame }}$ is the orientation angle of the mobile frame with respect to the global Earth fixed frame (Fig. 5). This angle can be easily obtained at the end of the mission from the evolution of the position of the two beacons.

Concerning the guidance and control of the AUV in the mobile network it should be noted that the onboard compass measures the orientation angle (yaw) of the AUV with respect to the local north, which is a global frame measurement. However, the execution of a mission defined in coordinates of 
the mobile frame requires the knowledge of the vehicle yaw angle in that frame, $\varphi_{r e f}$, which is given by

$$
\varphi_{\text {ref }}=\psi-\varphi_{\text {frame }} .
$$

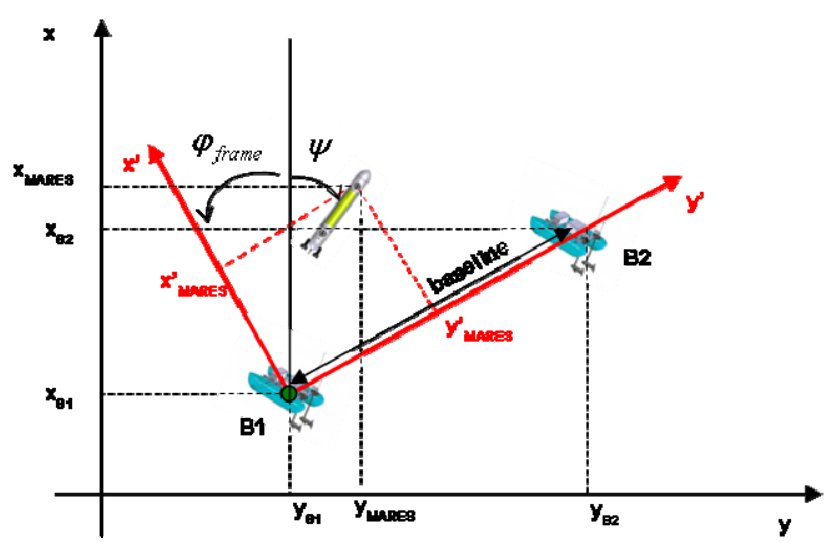

Figure 5. Global and mobile frame representation.

Since $\varphi_{\text {frame }}$ cannot be determined acoustically, we devised a Kalman filter to allow for its estimation is real time. In fact, this filter simultaneously estimates the AUV position in the mobile frame $\left(x^{\prime}, y^{\prime}\right)$, the orientation of the mobile frame $\varphi_{\text {frame }}$, and also the baseline length $b$. The filter integrates dead reckoning data, provided by a digital compass and by the motor controllers, with acoustic based positioning data composed by range measurements between the AUV and the beacons. The dynamics of the filter are characterized by

$$
\begin{aligned}
& \dot{x}^{\prime}=v \cos \left(\psi-\varphi_{\text {frame }}\right) \\
& \dot{y}^{\prime}=v \sin \left(\psi-\varphi_{\text {frame }}\right) \\
& \dot{\varphi}_{\text {frame }}=0 \\
& \dot{b}=0
\end{aligned}
$$

where $v$ corresponds to the vehicle speed, $\psi$ to the vehicle global orientation, $\varphi_{\text {frame }}$ to the mobile frame orientation and $b$ the baseline length.

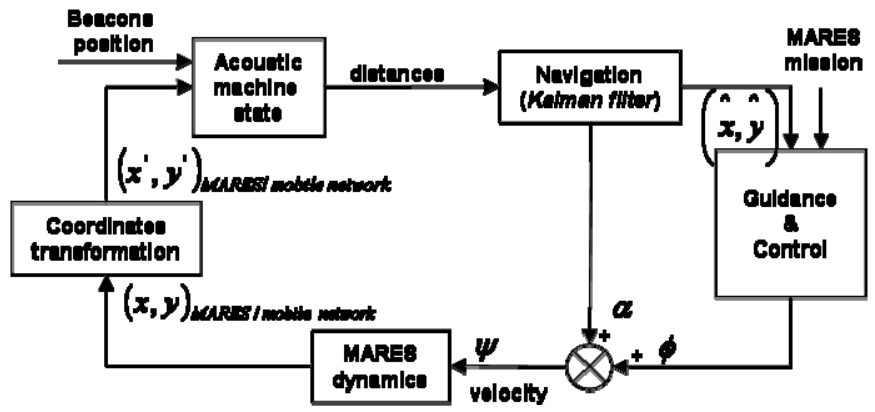

Figure 6. Simulation block diagram.

\section{SimUlATION RESUlts}

To simulate the navigation algorithm, the block diagram presented in Fig. 6 was implemented. Simple dynamic equations for the vehicle were considered, just for algorithms validation.

Several tests were performed with different scenario setup conditions, like different water currents, vehicle velocities, filters gains and trajectories, both for the mobile network and for the AUV.

The following results are from one experimental setup where it was assumed that one beacon is stopped at the origin and the other is drifting to west at $10 \%$ of the AUV velocity.

Figure 7 shows the evolution of the AUV in a global frame, which was obtained by the coordinate transformation given by equation (3).

Figure 8 shows the evolution of the AUV in the moving frame. Ideally it should be a perfect straight line. Nonetheless, the simulated trajectory is very close to that perfect line (note that the horizontal scale is largely amplified).

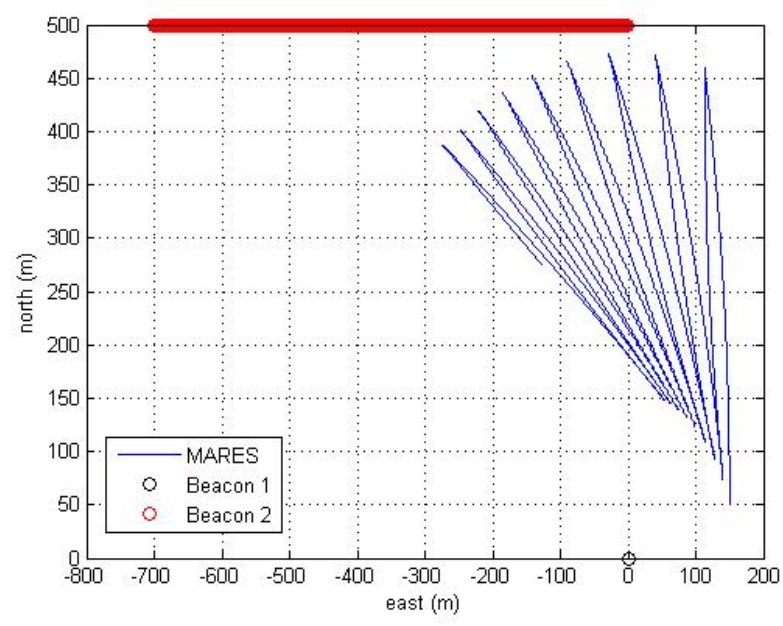

Figure 7. AUV and beacons global trajectories.

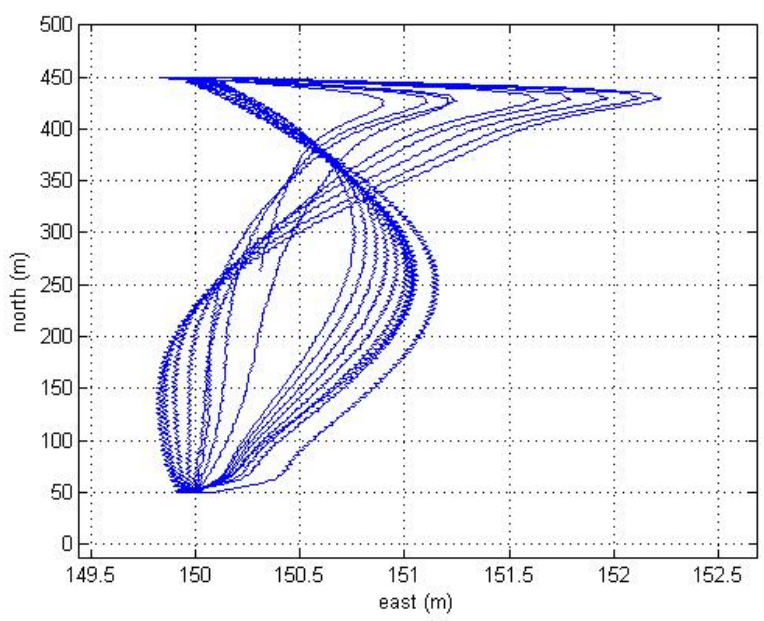

Figure 8. AUV trajectory in the mobile frame 


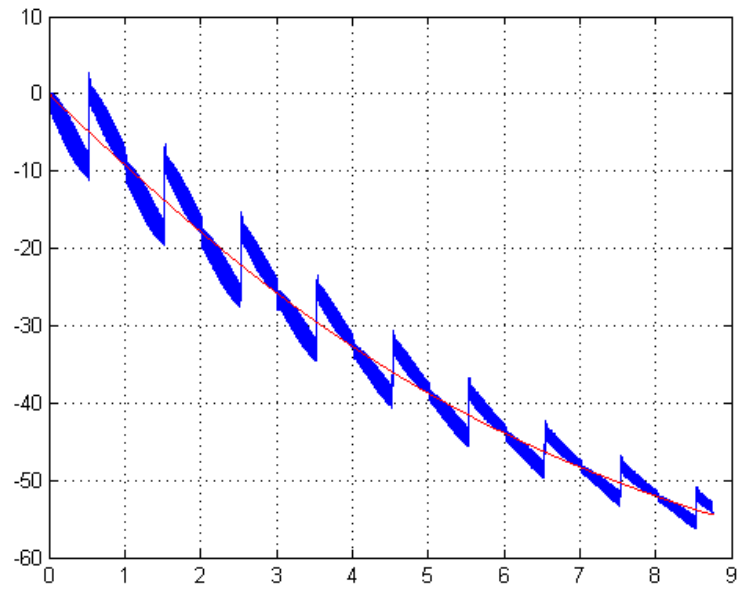

Figure 9. Real and estimated mobile frame orientation.

Figure 9 shows the mobile frame orientation and its estimate. The estimation error presents quite low values, somehow higher at the beginning of the simulation, corresponding to faster changes of the framework.

Other simulations, with both beacons moving with non constant velocity led to the conclusion that good estimation results are obtained as long as the beacons velocities are less than about $20 \%$ of the AUV MARES velocity.

\section{EXPERIMENTAL RESULTS}

The setup scenario of the experimental results was like the simulation scenario shown above. One beacon was kept fixed (beacon 2 in this case), and the other moved around, this time in several directions. This scenario allowed us to test the estimation of the most important variables, the baseline length, the mobile frame orientation and the MARES positioning.

During this experiments, MARES was programmed to go forward and backward along the line $(50,-50)$ to $(150,-50)$ as shown in Fig. 11.

The vehicle trajectory in the mobile frame, see Fig. 11, was kept near the defined line, with larger position errors near one end of the line. These increased errors are partially justified by the fact that the vehicle was navigating close to the baseline, due to the geometry of the operation area. Furthermore, the vehicle was using a way-point guidance algorithm and water currents were about $30 \%$ of the vehicle velocity, which negatively affects the performance of the vehicle guidance.

Figure 12 shows LBL ranges from MARES to the beacons. We can easily see some outliers, but the great majority of ranges are consistent.

Concerning the orientation of the mobile frame, there were quite large estimation errors (Fig. 13), but, nonetheless, the vehicle navigation had a satisfactory performance. This shows a low sensitivity of the navigation to such orientation errors, which is a good characteristic of the proposed solution.

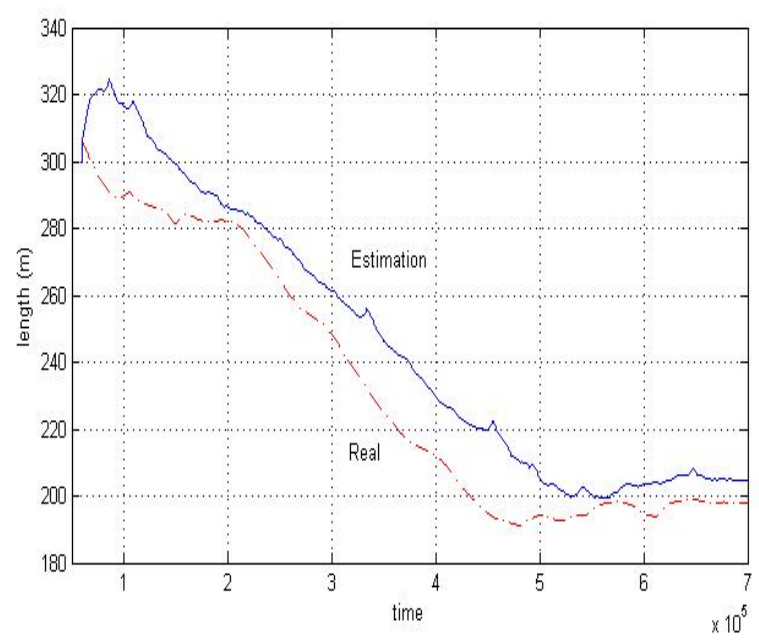

Figure 10. Baseline length.

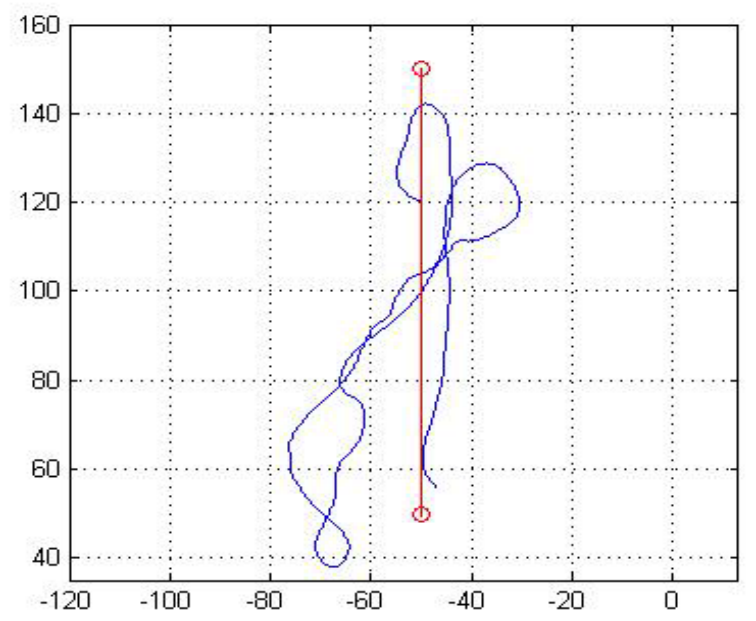

Figure 11. MARES trajectory in the mobile frame.



Figure 12. MARES measured beacons distance. 


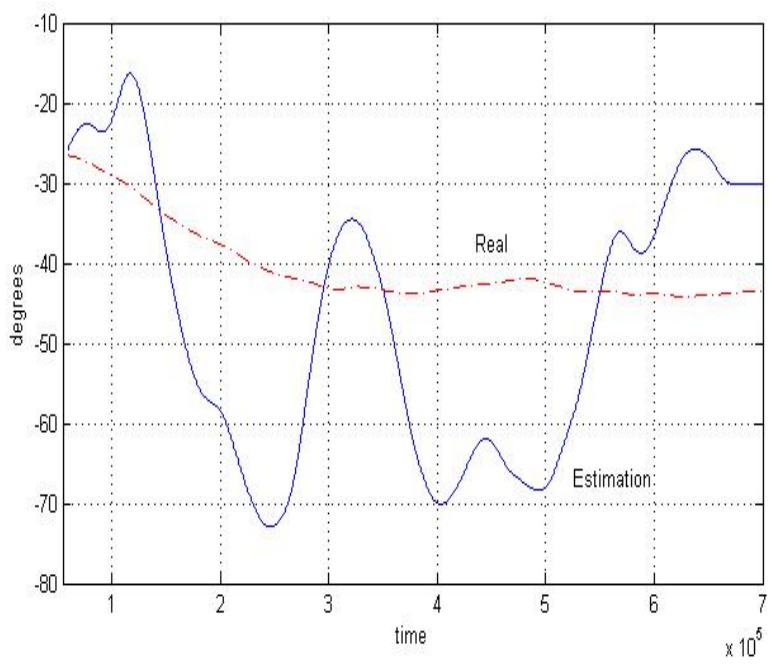

Figure 13. Mobile frame orientation.

Although some parameters of the navigation filter might required a finer calibration, these experimental results are quite promising, leaving space for a significant performance improvement.

\section{CONCLUSIONS}

Experimental results already proved that the proposed method for navigation in a mobile networks works. However, analyzing the experimental results and according to the vehicle characteristics, there are some possible changes to improve the navigation algorithm. One of the possible changes is to insert curve maneuvers between successive lines. During this transitions the vehicle adjusts is heading but that there is no update of the mobile frame orientation, since the proper estimation of this orientation requires that the vehicle is moving in a constant line with a constant speed.
Other possible improvement is the implementation of better line tracking algorithms. With a good line tracking algorithm, that compensates for water currents, minimizes the distance of the vehicle to the line, and makes the vehicle describe straighter lines, the navigation algorithm will certainly present a better performance.

The navigation of an AUV in a mobile network, allows for the real-time external control of the AUV trajectory, by the adequate modification of the mobile network. This is one of the major advances of mobile networks, allowing the definition of new mission paradigms.

\section{ACKNOWLEDGMENT}

This work was partially supported by the project INCORP --- Improved Navigation with Cooperative Robotic Platforms --- funded by FCT under Programa POSC (ref. POSC/EEA-SRI/59963/2004).

\section{REFERENCES}

[1] A. Matos, N.Cruz, A. Martins and F. L. Pereira. Development and implementation of a low-cost lbl navigation system for an AUV. In Proc. of the MTS/IEEE Conf. Oceans'99, Seatle, WA, USA. September 1999.

[2] N. Cruz, A. Matos, S. Cunha, S. Silva, "Zarco - An autonomous craft for underwater surveys", Proc. of Geomatica'2007, Barcelona, 2007.

[3] N. Cruz, A. Matos, "The MARES AUV, a Modular Autonomous Robot for Environment Sampling", Proc. of the MTS/IEEE Oceans'2008, Quebec, Canada, 2008.

[4] Nuno Santos. "Navigation of autonomous vehicle in a moving acoustic network". Master's thesis, Porto University, Faculty of Engineering, Portugal, 2008.

[5] Aníbal Matos and Nuno Cruz. "AUV navigation and guidance in a moving acoustic network". In Proc. of the MTS/IEEE Conf. Oceans'2005, Brest, France, June 2005. 